\title{
An analysis of perceptions from changes in optical size
}

\author{
WALTER C. GOGEL \\ University of California, Santa Barbara, California
}

\begin{abstract}
The allocation of perceived size and perceived motion or displacement in depth resulting from retinal size changes (changes in the visual angle of the stimulus) was investigated in situations in which all other cues of perceived changes in distance were absent. The allocation process was represented by the size-distance invariance hypothesis (SDIH), in which, for a given change in visual angle, the perceived depth was determined only by the amount of size constancy available. The changes in perceived size and perceived distance (perceived depth) were measured by kinesthetic observer (open-loop) adjustments in five situations. These situations consisted of optical expansions or contractions presented successively or simultaneously or as a mixture of successive and simultaneous presentations. The amounts of perceived motion or perceived displacement in depth obtained by kinesthetic measures were compared with those obtained from size constancy measures as applied to the SDIH. This latter measure accounted for more of the perceived depth obtained from simultaneous and mixed situations than it did for the perceived depth from the successive situations and more for the perceived depth obtained from the expansion than from the contraction situations, whether these were simultaneous or mixed. Perceived rigidity of the stimulus (perfect size constancy) clearly was not obtained in any of the situations. Significant partial size constancy and some predictive ability of the perceived sagittal motion was found using the SDIH in all the situations except in the successively presented contraction situation, with the predictive ability from the SDIH increasing with increases in the amount of size constancy. The difference between the observer's measures of the perceived motion or displacement in depth and the amount of perceived motion or displacement predicted from the perceptions of linear size using the SDIH is asserted to be due to a cognitive process associated with the perception of the different stimulus sizes as off-sized objects.
\end{abstract}

The depth cue of optical expansion or contraction (successive optical changes in size) and the depth cue of relative size or perspective (simultaneous optical changes in size) have some similar and some different characteristics. A similar characteristic is that in both situations the size of the stimulus on the eye changes systematically, producing perceptions of linear size and motion or displacement in distance even when the conditions are such that no other sources of information as to changing size or distance are available to the observer except the single variable of the changing visual angle of the stimulus. The perceived depth or perceived sagittal motion and the changes in perceived linear size resulting from the retinal changes or differences, under these circumstances, indicates that an increase in one of these perceptions often is accompanied by a decrease in the other. This can be described as a tradeoff between smaller changes in perceived linear size (an increasing amount of size constancy) and larger changes in perceived depth or perceived sagittal

The preparation of this article was supported by Research Grant MH 39457 from the U.S. National Institute of Mental Health to W.C.G. The author thanks Jerome D. Tietz for his assistance in the analysis of the data and suggestions concerning a previous draft and John M. Foley for helpful discussions on related issues. Correspondence should be addressed to W. C. Gogel, Department of Psychology, University of California, Santa Barbara, CA 93106 (e-mail: gogel@psych.ucsb.edu). motion. At the extremes of this tradeoff process, either one or the other of these two perceptions can be constant, with the other perception specified by all the change in retinal size (visual angle). For example, consider the case in which an object of constant physical size physically moves toward or away from the observer (an optical expansion or contraction) or a series of objects of the same physical size are distributed simultaneously in physical distance from the observer (the relative size or perspective cue of depth) with no cues other than the changes in visual angle available to the observer. If the physical situation is correctly perceived, the changes or differences in visual angle on the eye will result in no changes in the perceived linear size of the stimulus (complete size constancy, i.e., the veridical perception of linear size) with all the changes in visual angle responded to as veridical changes in perceived distance. Or, at the other extreme, the perception of linear size might vary proportionally to the changes in visual angle (zero size constancy), with the stimulus of changing size perceived as being at a constant physical distance. This tradeoff or division of the effect of the changes in visual angle between perceived linear size and perceived sagittal motion or displacement can be, and usually is, intermediate between these extremes, with the changing visual angle resulting in less than perfect linear size constancy and less perceived depth or sagittal motion than would be expected if the linear size con- 
stancy had been complete. According to this interpretation of the process of distributing inversely the effects of changes in visual angle between changes in perceived linear size and changes in perceived distance, the depth perceived should predict or be predicted by the amount of size constancy present. However, in the case of complete cycles of angular size expansion and contraction, it was found in one study (Swanston \& Gogel, 1986) that considerable perceived motion in depth occurred in the absence of any positive amount of size constancy. In another study (Gogel \& Eby, 1997), the amount of size constancy, although positive, was considerably smaller than was consistent with the amount of sagittal motion perceived by the observers. One purpose of the present study was to examine the relation between the amount of size constancy and the amount of sagittal motion or displacement in depth perceived by the observers by using presentations in addition to those in which only one stimulus is present at a time as in an optical expansion or contraction. Also, unlike the aforementioned two studies, in order to achieve a more complete analysis of the perceptual results of the optical changes, the perceptual effects of an expansion or contraction were examined by visually presenting repetitive half-cycles of expansions without contractions or repetitive half-cycles of contractions without expansions.

There are temporal presentation differences as well as process similarities between an expansion or contraction and the relative size or perspective cue. In an expansion or contraction, stimuli of the same shape but different in visual angle are presented successively either with continuous or discrete changes in visual angle. In the relative size or perspective cue, stimuli of the same shape but different in visual angle are presented simultaneously to produce either a continuous or a discrete relative size or perspective cue. It follows that the involvement of a memory factor is likely to differ in the different methods of presentation, thus possibly producing different allocations of changes in perceived linear size and changes in perceived distance in the different situations. The examination of the effect of this difference on the allocation process was the main purpose of the present study. Another purpose of the present study was to examine whether the prediction of the perceived motion or displacement in distance from the amount of size constancy available varies systematically between the different situations used in the study.

The size-distance invariance hypothesis (SDIH) provides a basis for expressing and comparing the relation between changes in perceived size (amount of size constancy) and perceived distance as a function of changes in the visual angle of the stimulus. The basic equation for this purpose using primed notation to indicate perceived variables and nonprimed to indicate physical variables is

$$
S^{\prime} / D^{\prime}=2 \tan (\theta / 2) .
$$

The application of Equation 1 to the beginning and ending (terminal) stimuli of a motion or displacement in perceived depth is

$$
d_{\mathrm{fn}}^{\prime}=S_{\mathrm{S}}^{\prime} / 2 \tan \left(\theta_{\mathrm{S}} / 2\right)-S_{\mathrm{L}}^{\prime} / 2 \tan \left(\theta_{\mathrm{L}} / 2\right)
$$

where $d_{\mathrm{fn}}^{\prime}$ is the perceived motion or displacement in depth between the farthest (f) and nearest (n) perceived distances of the stimuli, $S_{\mathrm{S}}^{\prime}$ and $S_{\mathrm{L}}^{\prime}$ are the perceived terminal linear sizes of the physically smallest and largest stimuli, and $\theta_{\mathrm{S}}$ and $\theta_{\mathrm{L}}$ are the smallest and largest physical sizes of the visual angles, respectively.

It will be noted that, consistent with Equation 2, as representative of the allocation process, for given changes in the physical visual angle, change in perceived linear size is the only variable available in the study to determine changes in perceived depth or perceived sagittal motion. The measured terminal values of $S_{\mathrm{S}}^{\prime}$ and $S_{\mathrm{L}}^{\prime}$ were used in this study to specify the amount of size constancy obtained in an optical size change. The greater the similarity between $S_{\mathrm{S}}^{\prime}$ and $S_{\mathrm{L}}^{\prime}$, the greater is the amount of size constancy present in the situation simulated by the optical change, and, using Equation 2 as representative of the $\mathrm{SDIH}$, the greater is the predicted $d_{\mathrm{fn}}^{\prime}$ (to be called the SDIH $d^{\prime}$ ). The amount of size constancy could be measured by either $S_{\mathrm{S}}^{\prime} / S_{\mathrm{L}}^{\prime}$ or by $S_{\mathrm{L}}^{\prime}-S_{\mathrm{S}}^{\prime}$. Defined in either of these ways, although not precisely predictive of SDIH $d^{\prime}$, the amount of size constancy is a monotonic function of the SDIH $d^{\prime} .^{1}$ In this study, the amount of size constancy usually will be expressed by $S_{\mathrm{S}}^{\prime} / S_{\mathrm{L}}^{\prime}$ rather than by $S_{\mathrm{L}}^{\prime}-S_{\mathrm{S}}^{\prime}$, and, as will be noted later, for the terminal values of $\theta_{\mathrm{S}}$ and $\theta_{\mathrm{L}}$ used in the optical size changes in the present study, $S_{\mathrm{S}}^{\prime} / S_{\mathrm{L}}^{\prime}$ is approximately an increasing linear function of the SDIH $d^{\prime}$. When $S_{\mathrm{S}}^{\prime} / S_{\mathrm{L}}^{\prime}=2 \tan \left(\theta_{\mathrm{S}} / 2\right)$ $2 \tan \left(\theta_{L} / 2\right)$, or 0.297 in the present study (a case of zero amount of size constancy), it is predicted from Equation 2 that the SDIH $d^{\prime}$ will be zero. When $S_{\mathrm{S}}^{\prime} / S_{\mathrm{L}}^{\prime}=1.000$ (perfect size constancy), it is expected from Equation 2 that the SDIH $d^{\prime}$ will be able to predict the total (nonzero) amount of perceived sagittal motion or displacement obtained. It will be noted that this concept of size constancy defined by $S_{\mathrm{S}}^{\prime} / S_{\mathrm{L}}^{\prime}$, as applied to a simulation of a physical sagittal motion or displacement, as in the present study, is not a measure of the perceived ratio of the terminal physical linear sizes produced on the monitor surface. Instead, it refers to the ratio of the perceived linear terminal sizes of the stimuli as simulated, not as produced on the monitor. Thus, for example, an $S_{\mathrm{S}}^{\prime} / S_{\mathrm{L}}^{\prime}$ of 1.00 would mean that the stimulus of constant linear size in the simulation was perceived as constant in linear size, whereas the physical size of the stimulus on the monitor would not be perceived as changing consistent with its changes in physical size. Equation 2 can be used to predict the SDIH $d^{\prime}$ that is expected in the simulation from the amount of obtained size constancy defined by $S_{\mathrm{S}}^{\prime}$ and $S_{\mathrm{L}}^{\prime}$. This predicted $d^{\prime}$ from the SDIH can then be compared with the $d^{\prime}$ as obtained from the observer's sagittal measures (labeled Obt. $d^{\prime}$ ) so as to evaluate the amount of perceived sagittal motion or displacement occurring in the simulation consistent with the SDIH.

In addition to the visual angle changes presented successively (optical expansion or contraction) or simultaneously (the relative size or perspective cue), another kind of 
situation intermediate between the successive and simultaneous presentations was also used in the present study. In this situation, stimuli of the same shape but different retinal sizes were presented successively, but, once a stimulus of a particular visual angle was presented, it continued to be visible during the remaining presentations in that sequence. These three kinds of optical size changes or differences and their subdivision in terms of expansion or contraction are illustrated schematically in Figure 1.

Figure 1 represents situations in which stimuli of changing visual angle are presented at a constant distance from the observer on a physically stationary monitor screen. The symbols T1-T4 specify the temporal order in which the stimuli were presented on the monitor and were viewed by the observer. In order to reduce the size of the drawings, only four presentations (T1-T4) of the seven presentations actually used in each sequence in the study are shown in Figure 1. Unlike the drawings of Figure 1, all of the square stimuli in all of the situations were presented with the center of the stimulus always at the same physical position on the monitor. Situations Ie and Ic of Figure 1 illustrate successive discrete presentations of stimuli that produce an optical expansion and an optical contraction, respectively. The expected perception of motion in depth from the simulation is that of a square moving toward the observer in the case of the optical expansion (Ie), and a square moving away from the observer in the case of the optical contraction (Ic). Situation III illustrates the same differences in visual angle between the squares as in Situations Ie or Ic, but in Situation III all the sizes of the stimulus squares are simultaneously visible in the presentations T1-T7. The perception simulated in Situation III is that of the repetitive presentations of the same constant configuration of squares with the unchanging stimulus configuration in each presentation perceptually extended the same amount in depth. In Situations IIe and IIc, the same amount of optical change is represented between the stimulus squares, as in Situations I and III. However, as in Situations Ie and Ic, and unlike Situation III, a new square of different size is shown as the presentation changes from T1-T7. But, unlike Situations Ie and Ic, and like Situation III, in Situations IIe and IIc a stimulus of a particular visual angular size, upon being presented, continues to be present throughout the remainder of the series. The perception simulated in Situations IIe and IIc is that of a configuration of stimuli growing in number and thereby increasing in its depth extent as the number of presentations increases from $T 1$ to $T 7$. In Situation IIe, the simulated growth in depth is toward the observer (a form of optical expansion), and in Situation IIc, the simulated growth in depth is away from the observer (a form of optical contraction). Situations Ie and Ic will be called pure optical size changes (a pure optical expansion or pure optical contraction, respectively) since the stimuli of different sizes are always presented successively. Situation III will be called a pure relative (perspective) size cue since all of the stimuli always are shown on each presentation. Situations IIe and IIc will be called mixed optical changes (a mixed optical expansion or a mixed optical contraction, respectively), since they have similarities and differences with respect to both Situations I and III. Since the stimulus sizes and differences in the stimulus sizes present in all the situations of Figure 1, as used in the present experiments, were the same, the perceptions of linear size and changes or differences in perceived linear size and the extent of perceived sagittal motion or depth produced by the different situations can be meaningfully compared.

\section{EXPERIMENT 1}

Experiment 1 measured the perceived linear size and the perceived depth generated by the sequence of presentations of the kind illustrated in Situations I and II of Figure 1. However, unlike the conditions represented by Figure 1, seven rather than four successive presentations, $\mathrm{T} 1, \mathrm{~T} 2, \mathrm{~T} 3, \mathrm{~T} 4, \mathrm{~T} 5, \mathrm{~T} 6$, and T7, were used throughout this study.

\section{Method}

Observers. The observers were 12 graduate students from the psychology department who were paid for participating in the experiment. All had a Landolt ring acuity of at least $20 / 30$ in the right eye, corrected if necessary as measured on a Keystone Orthoscope. None were informed regarding the purpose of the experiment.

Apparatus. The observer sat on a stool adjustable in height in an enclosed observation booth with his/her head in a physically stationary chinrest located in front of a large viewing aperture with the viewing aperture positioned at the front of an alley $36 \mathrm{~cm}$ wide extending from the observer's position to beyond the distance of the stimulus. The viewing aperture was used to present or occlude the observer's view of the stimulus. The stimuli throughout this study were always viewed monocularly with the left eye of the observer covered by an eye patch. The viewing aperture and the walls and floor of the alley were covered with black cloth so that when the observer was viewing the stimulus from a dark observation booth nothing was visible except the stimulus. The stimuli were generated on a Conrac model SNA/23C black-and-white composite video monitor by an IBM PC computer using a CGA-compatible video display board. The display screen was located in the alley always positioned in the observer's frontal plane $40.0 \mathrm{~cm}$ from the observer's right eye with the center of the stimulus at eye level for all presentations. The stimuli, consisting of seven concentric outline luminous squares with horizontal and vertical sides, always were presented in the totally dark surround. Only Situations Ie, Ic, Ile, and IIc of Figure 1 were used in Experiment 1. The widths of the squares on the monitor screen from smallest to largest in the situations of Experiment 1 and in all the sequences throughout the study were $6.6,7.6,8.7,10.2,12.6,16.1$, and $22.2 \mathrm{~cm}$. The luminous lines with which they were constructed were approximately $0.5 \mathrm{~mm}$ wide. The changes in size between the squares of a sequence in Experiment 1 were designed to simulate a motion in sagittal depth (Situations Ie and Ic) or a configuration expanding in sagittal depth (Situations IIe and IIc) so that the increments of simulated depth motion or depth growth, whether in Situation I or II, were constant within an expansion or within a contraction.

The observers had two tasks that used open loop procedures. One was to indicate the perceived lateral (linear) size of the perceptually farthest (physically smallest) and perceptually nearest (physically 


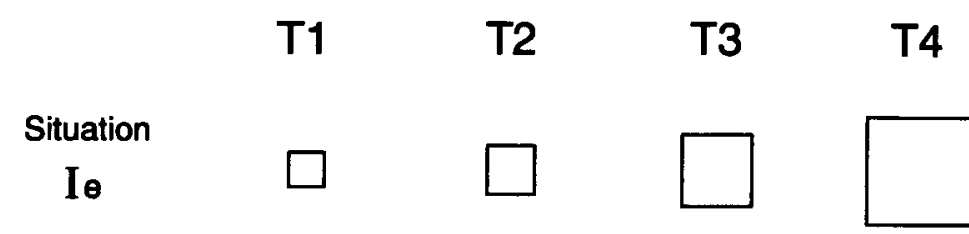

Successive presentations (T1 to T4) of an optical expansion, to be called a "pure optical expansion."
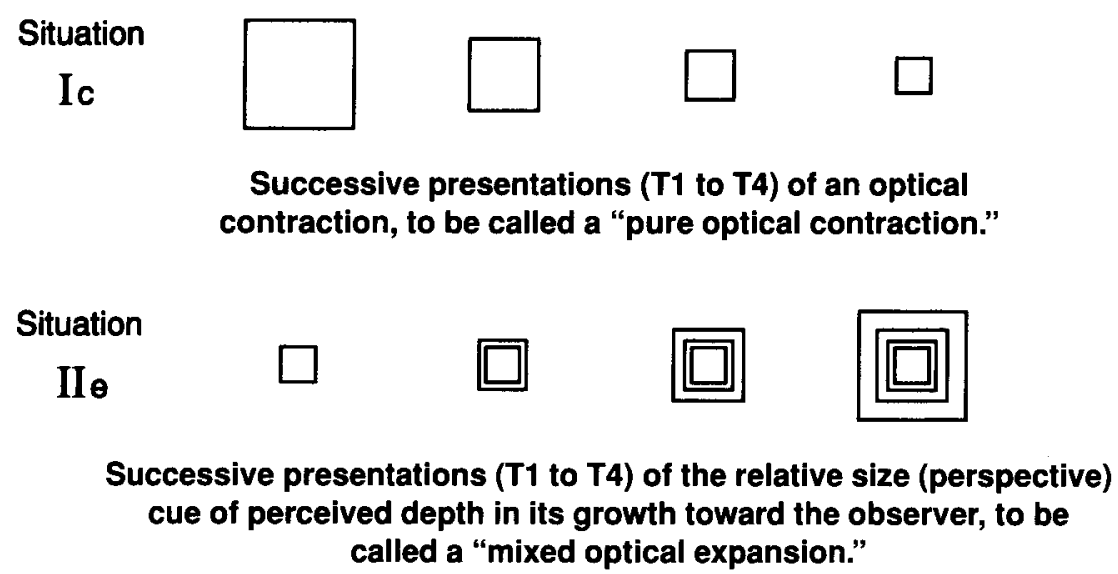

Situation
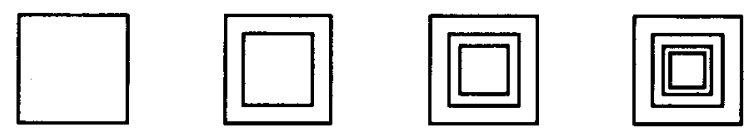
Successive presentations ( $\mathrm{T} 1$ to $\mathrm{T4}$ ) of the relative size (perspective) cue of perceived depth in its growth away from the observer, to be called a "mixed optical contraction."

\section{Situation \\ III}
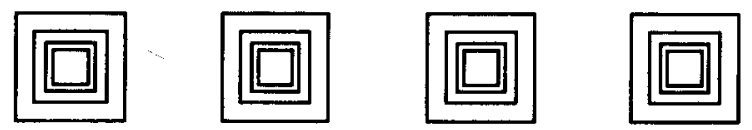

Successive (repetitive) presentation (T1 to T4) of the relative size (perspective) cue of perceived depth, to be called a

"pure relative size (perspective) cue."

Figure 1. Schematic illustration of the series of successive presentations of the stimuli used to provide the optical changes. The stimuli were displayed on a monitor screen at a constant distance and direction from the observer with the center of all the stimuli straight ahead of the observer. The numbers accompanying the $T s$ in the drawing indicate the order in which the stimuli were presented. To avoid an excessively large drawing, only four successive presentations (T1-T4) are shown, rather than the seven successive presentations (T1-T7) that were used throughout the study. The optical size changes throughout the study from the smallest to the largest stimulus were always the same for all the situations. Subscripts $e$ and $c$ indicate an expected perceived expansion and contraction, respectively, of stimuli during the presentations. Situations Ie, Ic, IIe, and IIc were used in Experiment 1, and Situations IIe, IIc, and III were used in Experiment 2.

largest) stimulus in each series of presentations. The other was to indicate the perceived sagittal motion, the perceived growth in depth, or the perceived displacement occurring between the perceptually farthest and perceptually nearest stimulus in each series of presentations. To obtain measures of perceived lateral size, a response device was used consisting of two sliding metal posts lo- cated below the alley floor so as not to be visible to the observer, with a small knob on top of each post. This pair of laterally movable posts was used to measure the perceived linear sizes of the two terminal stimuli. The posts were $1.25 \mathrm{~cm}$ square in cross-section and $10.0 \mathrm{~cm}$ in length and were pinned at a constant distance beneath the alley floor, approximately $4.0 \mathrm{~cm}$ forward of the ob- 
server's eye, with their tops $30.0 \mathrm{~cm}$ below eye level. The observer, using unseen hands (the left hand grasping the left post and the right hand grasping the right post), simultaneously separated the unseen posts (knobs), laterally using kinesthesis until their separation indicated the perceived linear size of the stimulus. The lateral separation of the posts adjusted in this manner was recorded on a computer by means of potentiometers interfaced to an $A / D$ board with an 8-bit resolution. The largest possible physical lateral separation using this apparatus was $47.3 \mathrm{~cm}$. To obtain measures of perceived sagittal motion or sagittal displacement of the two terminal stimuli, the two separable posts were brought together and were unpinned so as to be movable sagitally by the observer as a single indicator. An additional physically stationary post protruding $10.0 \mathrm{~cm}$ downward from the underside of the alley floor and located $3.0 \mathrm{~cm}$ forward of the observer's eyes formed a stationary reference point for the measurement of the sagittal depth perceived in each series of presentations. The observer indicated the perceived amount of sagittal motion or displacement of the stimuli at their terminal distances by adjusting with unseen hands kinesthetically (the left hand on the near stationary post and the right hand on the movable pair of posts) until the sagittal separation of the inner edges of the unseen stationary post and movable pair of posts equaled the depth of motion or displacement as visually perceived. The largest possible physical sagittal separation of the posts using this apparatus was $83.5-3.0=80.5 \mathrm{~cm}$. The sagittal separation adjusted by the observer using this open-loop procedure also was recorded on the computer using an additional potentiometer.

Procedure. In the optical expansion or contraction of Situation Ie or Ic, the squares were presented sequentially either from the smallest to the largest stimulus (an expansion) or from the largest to the smallest stimulus (a contraction). A square appeared for $0.44 \mathrm{sec}$ (except that the first and last stimulus were visible for $2.5 \mathrm{sec}$ ) and then was immediately replaced with the next square in the series. That is, throughout the study, whenever an increment of size change was added to or subtracted from the next presentation in the sequence, it occurred instantly (in jumps). In the mixed expansion or contraction (Situation IIe or IIc of Figure 1), the same sequence and durations of successive presentations occurred as in the expansion or contraction sequence of Situation I, but in Situation II each of the squares on the monitor, once having appeared, continued to appear in the following presentations of the sequence until the presentation of the last square in that series. In the presentation of the last square of the series, all seven squares were visible for $2.5 \mathrm{sec}$. The expansion or contraction for each sequence, whether a pure or a mixed optical expansion or contraction on a given trial, was in only one direction; that is, the simulated motion or extension was either toward the observer (an expansion) or away from the observer (a contraction) with no return in the opposite direction. The series of these half-cycle presentations was repeated a number of times depending on how long it took the observer to indicate separately the perceived linear sizes $\left(S_{\mathrm{S}}^{\prime}\right.$ and $\left.S_{\mathrm{L}}^{\prime}\right)$ and the perceived sagittal motion or displacement (Obt. $d^{\prime}$ ). Between trials, the observers were light adapted by viewing a lighted surface. Before the experiment began, in addition to verbal instructions, the lateral adjustment procedure was illustrated for the observer using a small model of the lateral adjustment posts and a stimulus. In the experimental observation booth the observer was given some practice in moving the sagittal and lateral adjustment posts in the absence of a stimulus. Also, the observer was instructed, "In all of the tasks we are interested only in what you perceive and not in the physical conditions you think might be present, if these differ."

In addition to the series of dynamic presentations illustrated in Figure 1, the first stimulus presented in an expansion or contraction in Situation I or II also was presented separately (statically) prior to the first time that the particular expansion or contraction was presented to the observer. Thus, $7.5 \mathrm{sec}$ before the presentation of the first stimulus of an expansion series (Situation Ie or IIe), the smallest stimulus (the 6.6-cm square) was presented alone (singly) and the observer indicated its perceived lateral (linear) size by the kinesthetic lateral separation of the unseen measuring posts and hands. Also, before the presentation of the first stimulus of the contraction series (Situation Ic or IIc), the largest stimulus (22.2-cm square) was presented alone (singly), and its perceived lateral (linear) size was also measured by the kinesthetic lateral separation of the unseen measuring posts and hands. The purpose of presenting the single (static) outline square, 6.6 or $22.2 \mathrm{~cm}$ wide, prior to the beginning of a series of expansions or contractions, respectively, was to compare the amount of size constancy $\left(S_{\mathrm{S}}^{\prime} / S_{\mathrm{L}}^{\prime}\right)$, if any, obtained between the 6.6- and $22.2-\mathrm{cm}$ single (static) stimulus with that obtained when the same two stimuli were the terminal sizes of the series of seven stimuli. If the amount of size constancy is essentially the same from these two conditions, it might be that the apportionment occurring in the series of presentations is related to the perceived size occurring in the two single static stimuli of different sizes, in which each stimulus was presented at substantially different times. After the $7.5 \mathrm{sec}$ of darkness, the appropriate series of dynamic presentations began with either a pure optical expansion or contraction or a mixed optical expansion or contraction. Under each of the dynamic conditions three responses were obtained: (1) The perceived linear size, $S_{\mathrm{S}}^{\prime}$, of the perceptually most distant (physically smallest) square was measured using the laterally movable posts; (2) the perceived linear size, $S_{\mathrm{L}}^{\prime}$, of the perceptually closest (physically largest) square was measured using the laterally movable posts; and (3) the perceived sagittal depth (Obt. $d^{\prime}$ ) between the perceptually nearest and perceptually farthest squares was measured by kinesthetically adjusting the sagittal separation between the physically stationary post and the sagitally movable posts, which for that task were laterally merged. After each response was recorded, $4.0 \mathrm{sec}$ of total darkness elapsed before the same situation was again presented and the next measure of either $S_{\mathrm{S}}^{\prime}, S_{\mathrm{L}}^{\prime}$, or perceived sagittal motion or displacement, was obtained. The order of these three responses in the dynamic situations (Ie, Ic, IIe, and IIc) measured on consecutive trials was always the same for a given observer but was varied between observers in such a way that half of the observers first measured the perceived depth before measuring the two terminal perceived sizes, with the reverse order used with the remaining observers. The two measures of terminal perceived linear size were never separated by a measure of perceived sagittal motion or displacement in depth. For the two perceived terminal size responses, half the observers measured the perceived linear size of the perceptually most distant stimulus first while the other half used the reverse order. The order of the four dynamic situations-pure optical expansion, mixed optical expansion, pure optical contraction, and mixed optical contraction - was counterbalanced between observers so that the first situation was expansion for half the observers and contraction for the other half, with the order of presenting the pure and mixed optical changes counterbalanced in a similar manner.

\section{Results}

The perception of linear size. The results from Experiment 1 are shown in Table 1. The left two columns of Table 1 contain the average left-to-right extent of the perceived linear sizes of the smallest, $S_{S}^{\prime}(6.6 \mathrm{~cm}$ wide $)$, and largest, $S_{L}^{\prime}$ ( $22.2 \mathrm{~cm}$ wide), stimuli as obtained from Situations Ie, Ic, IIe, and IIc. The third column contains the average $S_{\mathrm{S}}^{\prime} / S_{\mathrm{L}}^{\prime}$ as determined from the distribution of $S_{\mathrm{S}}^{\prime} / S_{\mathrm{L}}^{\prime}$ values. Size constancy can be estimated by either the average ratio, $S_{\mathrm{S}}^{\prime} / S_{\mathrm{L}}^{\prime}$, or the average difference, $S_{\mathrm{L}}^{\prime}-$ $S_{\mathrm{S}}^{\prime}$. Using the average ratio of $S_{\mathrm{S}}^{\prime} / S_{\mathrm{L}}^{\prime}$ as the measure of 
Table 1

Average Results from Experiment 1

\begin{tabular}{|c|c|c|c|c|c|c|c|}
\hline & \multicolumn{4}{|c|}{ Pure Optical Expansion, Ie } & \multirow[b]{2}{*}{$\operatorname{SDIH} d^{\prime}$} & \multicolumn{2}{|c|}{ Single Presentation } \\
\hline & $S_{\mathrm{S}}^{\prime}$ & $S_{\mathrm{L}}^{\prime}$ & $S_{\mathrm{S}}^{\prime} / S_{\mathrm{L}}^{\prime}$ & Obt. $d^{\prime}$ & & $S_{\mathrm{S}}^{\prime}$ & $D^{\prime}$ from $S_{\mathrm{S}}^{\prime}$ \\
\hline \multirow{4}{*}{$\begin{array}{l}M \\
S D\end{array}$} & $9.6 \dagger$ & $27.0+$ & $0.365^{*}$ & $17.1 \dagger$ & $9.3^{*}$ & $10.5 \dagger$ & $63.4 \dagger$ \\
\hline & 2.7 & 8.0 & 0.081 & 5.0 & 13.7 & 2.5 & 15.2 \\
\hline & \multicolumn{4}{|c|}{ Pure Optical Contraction, Ic } & & \multicolumn{2}{|c|}{ Single Presentation } \\
\hline & $S_{S}^{\prime}$ & & $S_{\mathrm{S}}^{\prime} / S_{\mathrm{L}}^{\prime}$ & Obt. $d^{\prime}$ & $\operatorname{SDIH} d^{\prime}$ & & $D^{\prime}$ from $S_{\mathrm{L}}^{\prime}$ \\
\hline \multirow{4}{*}{$\begin{array}{l}M \\
S D\end{array}$} & $\overline{8.6 \dagger}$ & $28.3 \dagger$ & 0.312 & $22.2 \dagger$ & 0.9 & $\overline{28.4 \dagger}$ & $512 \div$ \\
\hline & & 7.0 & 0.084 & 7.2 & 13.4 & 7.0 & 12.7 \\
\hline & \multirow{2}{*}{\multicolumn{4}{|c|}{$\begin{array}{l}\text { Mixed Optical Expansion, Ile } \\
S_{\mathrm{S}}^{\prime} \quad S_{\mathrm{L}}^{\prime} \quad S_{\mathrm{S}}^{\prime} / S_{\mathrm{L}}^{\prime} \quad \text { Obt. } d^{\prime}\end{array}$}} & & \multicolumn{2}{|c|}{ Single Presentation } \\
\hline & & & & & SDIH $d^{\prime}$ & $S_{\mathrm{S}}^{\prime}$ & $D^{\prime}$ from $S_{\mathrm{S}}^{\prime}$ \\
\hline \multirow{4}{*}{$\begin{array}{l}M \\
S D\end{array}$} & $9.4 \dagger$ & $23.9+$ & $0.403 \dagger$ & $16.8 \dagger$ & 14. & $9.6+$ & 58 \\
\hline & 3.1 & 7.1 & 0.091 & 5.6 & 13.2 & 2.5 & \\
\hline & \multicolumn{5}{|c|}{ Mixed Optical Contraction, IIc } & \multicolumn{2}{|c|}{ Single Presentation } \\
\hline & & & & Obt. $d^{\prime}$ & $\operatorname{SDIH} d^{\prime}$ & $S_{\mathrm{L}}^{\prime}$ & \\
\hline$M$ & $9.3 \dagger$ & $28.2 \dagger$ & 0.338 & $21.3 \dagger$ & 5.4 & $28.4 \dagger$ & $51.2 \dagger$ \\
\hline$S D$ & 2.2 & 7.4 & 0.063 & 6.3 & 10.6 & 6.7 & 12.2 \\
\hline
\end{tabular}

Note-All the values of $S^{\prime}, d^{\prime}$, and $D^{\prime}$ are in centimeters. The values of $S_{\mathrm{S}}^{\prime} / S_{\mathrm{L}}^{\prime}$ are the averages of the ratios of the distributions of $S_{\mathrm{S}}^{\prime} / S_{\mathrm{L}}^{\prime}$, not the ratio of the average values of $S_{\mathrm{S}}^{\prime}$ and $S_{\mathrm{L}}^{\prime}$ shown in the previous two columns. The statistically significant differences are from zero for all of the means except those labeled $S_{\mathrm{S}}^{\prime} / S_{\mathrm{L}}^{\prime}$, in which the significant differences are from zero constancy, where zero constancy is $2 \tan$ $\left(\theta_{\mathrm{S}} / 2\right) / 2 \tan \left(\theta_{\mathrm{L}} / 2\right)$, or 0.297 in the present study. The values of perceived egocentric distance $\left(D^{\prime}\right)$ for the single (static) presentations were computed using Equation 1. ${ }^{*} p<.05 . \quad{ }^{\dagger} p<.01$.

size constancy, the range of size constancy is from zero constancy at 0.297 (e.g., $6.6 \mathrm{~cm} / 22.2 \mathrm{~cm}$ ) to perfect constancy at 1.00 (e.g., $6.6 \mathrm{~cm} / 6.6 \mathrm{~cm}$ for an optical expansion or $22.2 \mathrm{~cm} / 22.2 \mathrm{~cm}$ for an optical contraction). Size constancy as measured by either of these methods is expected to be related to the $d_{\mathrm{fn}}^{\prime}$ predicted from Equation 2, since, in addition to the given values of the terminal visual angles, the values of $S_{\mathrm{S}}^{\prime}$ and $S_{\mathrm{L}}^{\prime}$ are the only variables in the computation of $d_{\mathrm{fn}}^{\prime}$ using Equation 2 of the SDIH. In order of increasing magnitude, the linear size constancy using the average $S_{\mathrm{S}}^{\prime} / S_{\mathrm{L}}^{\prime}$ data of Table 1 was 0.312 from Situation Ic, 0.338 for Situation IIc, 0.365 for Situation Ie, and 0.403 for Situation IIe. The corresponding size constancy results, in order of decreasing magnitude, using the difference measure $\left(S_{\mathrm{L}}^{\prime}-S_{\mathrm{S}}^{\prime}\right)$, are $19.7 \mathrm{~cm}$ for Situation Ic, $18.9 \mathrm{~cm}$ for Situation IIc, $17.4 \mathrm{~cm}$ for Situation Ie, and $14.5 \mathrm{~cm}$ for Situation IIe. The results from both measures indicate that the amount of size constancy, although quite small throughout the experiment, was greater (increasing values in the direction of unity in the case of $S_{\mathrm{S}}^{\prime} / S_{\mathrm{L}}^{\prime}$ and decreasing values in the direction of zero in the case of $S_{\mathrm{L}}^{\prime}-S_{\mathrm{S}}^{\prime}$ ) in the expansions (le and IIe) than in the contractions (Ic and IIc), and was somewhat greater in the mixed optical situations (IIe and IIc) than in the comparable pure optical situations (Ie and Ic).

A three-factor analysis of variance (ANOVA) was completed on the $S^{\prime}$ data in the left two columns of Table 1 for the effects of (A) pure optical size change versus mixed optical size change, (B) expansion versus contraction, and $(\mathrm{C})$ small versus large terminal stimulus. Only the $\mathrm{C}$ factor and the interaction of $\mathrm{AC}$ and $\mathrm{BC}$ were statistically significant at, at least, the .05 level $[F(1,11)=126.95, p<$ $.01, F(1,11)=6.22, p<.05$, and $F(1,11)=12.87, p<.01$, respectively]. The interactions indicate that size constancy, as measured in this case by the difference method, varied significantly as a function of both Factors $A$ and B. A two-factor ANOVA was completed on the average $S_{\mathrm{S}}^{\prime} / S_{\mathrm{L}}^{\prime}$ data of Table 1 for the effect of (A) size ratios from pure versus mixed and (B) expansion versus contraction. Both the A and B factors were statistically significant $[F(1,11)=8.370, p<.02$, and $F(1,11)=9.147, p<.02$, respectively]. These effects indicate that size constancy as measured by the ratio method varied significantly in the different situations.

The average perceived linear size $\left(S_{S}^{\prime}\right.$ or $\left.S_{\mathrm{L}}^{\prime}\right)$ of the single (static) stimulus of the same size as the starting size of the pure or mixed dynamic optical changes is shown in the right portion of Table 1 . The difference in the perceived size of the small $\left(S_{\mathrm{S}}^{\prime}\right)$ and large $\left(S_{\mathrm{L}}^{\prime}\right)$ stimulus of the static presentations averaged over the experiment, as expected, was statistically significant $[t(11)=10.27, p<$ $.01]$. From the perceived linear size of the static stimuli using Equation 1, the perceived egocentric starting distance, $D^{\prime}$, of the first (starting) presentation in the series of optical changes was computed, with the results shown in the far-right column of Table 1 under the headings $D^{\prime}$ from $S_{\mathrm{S}}^{\prime}$ and $D^{\prime}$ from $S_{\mathrm{L}}^{\prime}$. According to these computed values, the perceived egocentric distance of the single static stimulus, whether the static linear size was small $\left(S_{\mathrm{S}}^{\prime}\right)$ or large $\left(S_{\mathrm{L}}^{\prime}\right)$, was greater than the 40.0 -cm physical distance. In this study only the rather weak cue of accommodation (and accommodative convergence) was available to localize the perceived egocentric distance of the single static stimulus at the physical distance of $40 \mathrm{~cm}$. Perceived egocentric distances larger than $40 \mathrm{~cm}$, however, are expected from the specific distance tendency (SDT) for both the small and the large static stimuli. The SDT is the tendency in the absence of effective cues of egocentric distance for stimuli to appear at several meters from the observer, with the resulting perceived egocentric distance a compromise between the SDT and whatever weak distance cues remain (Gogel, 1969; Mershon, Jones, \& Taylor, 1993).

The perceived egocentric distance of the small (static) single presentation was significantly larger than the perceived egocentric distance of the large (static) single presentation averaged over the experiment as computed from the perceived linear sizes using Equation $1[t(11)=$ $2.25, p<.05]$. The importance of this result is to indicate that the perception of the initial starting stimulus of the contraction situations was at a distance closer to the observer than the perception of the initial starting distance of the expansion stimulus, with a difference of $9.6 \mathrm{~cm}$ (i.e., $60.8 \mathrm{~cm}-51.2 \mathrm{~cm}$ ). The overall average perceived linear sizes of the small and the large single stimuli in Experiment 1 (10.1 and $28.4 \mathrm{~cm}$, respectively) are similar to but slightly larger than the terminal overall perceived linear sizes in the series of presentations $(9.2$ and $26.8 \mathrm{~cm}$, 
respectively). Consistent with the difference in the perceived egocentric distances of the small and the large stimuli in the single static presentations, the overall size constancy in the single static presentations of Experiment 1 defined by the ratio of the average values of $S_{\mathrm{S}}^{\prime}$ and $S_{\mathrm{L}}^{\prime}$ is 0.354 , which is significantly larger than the value of 0.297 , which would have indicated a size constancy of zero $[t(11)=2.49, p<.05]$.

The presence of a significant amount of size constancy as calculated from the perceived sizes in both the static and the dynamic conditions suggests that the amount of size constancy obtained from the static stimuli may have contributed to the amount of size constancy present in the dynamic conditions. Or, because different observers had different orders of the different dynamic situations, the perceived sizes from the dynamic series may have influenced the presence of an amount of size constancy between the static presentations.

The perception of depth. The average data from the sequences of presentations concerning the perceived sagittal motion or displacement (growth) in depth are shown in two columns of Table 1 . The column labeled Obt. $d^{\prime}$ gives the average results from the kinesthetic sagittal adjustments of the posts to measure the perceived motion in depth from the pure optical size changes (Situations Ie and Ic) and the perceived growth in depth of the mixed optical size changes (Situations IIe and IIc). The average values of Obt. $d^{\prime}$ from the expansions, pure or mixed ( 17.1 and $16.8 \mathrm{~cm}$, respectively), are less than the averages from the contractions, pure or mixed (22.2 and $21.3 \mathrm{~cm}$, respectively). However, the average values of Obt. $d^{\prime}$ from the pure optical size changes, expansion or contraction (17.1 and $22.2 \mathrm{~cm}$, respectively), are similar to the averages from the mixed optical change, expansion or contraction ( 16.8 and $21.3 \mathrm{~cm}$, respectively). These comparisons of the Obt. $d^{\prime}$ values from the experiment are supported by a two-factor ANOVA using the factors of pure versus mixed optical change and expansion versus contraction. Only the expansion versus contraction factor was statistically significant at, at least, the 0.5 level $[F(1,11)=7.91, p<.02]$ in Experiment 1 .

The column labeled SDIH $d^{\prime}$ in Table 1 gives the average values of $d^{\prime}$ calculated by substituting the obtained $S_{\mathrm{S}}^{\prime}$ and $S_{\mathrm{L}}^{\prime}$ results in Equation 2 of the SDIH for each observer with $2 \tan \left(\theta_{\mathrm{S}} / 2\right)=0.165$ and $2 \tan \left(\theta_{\mathrm{L}} / 2\right)=0.555$. The values of $d^{\prime}$ calculated in this manner indicate the amount of perceived depth that can be accounted for by the SDIH as expressed by Equation 2, in which $S_{\mathrm{S}}^{\prime}$ and $S_{\mathrm{L}}^{\prime}$ are the only perceptual situational variables. It will be noted that these values of $d^{\prime}$ (i.e., SDIH $d^{\prime}$ ) usually are considerably less than the $d^{\prime}$ as measured by the kinesthetic sagittal adjustments (i.e., Obt. $d^{\prime}$ ) in the four series of presentations of optical change. These differences between Obt. $d^{\prime}$ and SDIH $d^{\prime}$ must be explained by processes other than the amount of size constancy available as expressed through the SDIH. In the case of pure optical expansion (Ie), the unaccounted for $d^{\prime}$ is $17.1-9.3$, or $7.8 \mathrm{~cm}$. In the case of pure optical contraction (Ic), it is $22.2-0.9=21.3 \mathrm{~cm}$. In the case of mixed optical expansion (IIe), it is $16.8-14.0=2.8 \mathrm{~cm}$, and in the case of mixed optical contraction (IIc), it is $21.3-5.4=$ $15.9 \mathrm{~cm}$. The SDIH accounted for considerably more of the perceived motion or growth in depth in the optical expansions (Ie and IIe) than in the optical contractions (Ic and IIc) and more in the mixed optical change (IIe and IIc) than in the pure optical change (Ie and Ic).

An ANOVA was completed on the Obt. $d^{\prime}$ minus the SDIH $d^{\prime}$ computed from Equation 2 using the factors of pure optical change versus mixed optical change and expansion versus contraction. Both factors were statistically significant $[F(1,11)=9.25, p<.01$, and $F(1,11)=14.35$, $p<.01$, respectively].

It also seems clear that the computed values of SDIH $d^{\prime}$ in Table 1 differed substantially between the different situations. The average values of SDIH $d^{\prime}$ calculated from the expansions, pure or mixed $(9.3$ and $14.0 \mathrm{~cm}$, respectively), are larger than the averages calculated from the contractions, pure or mixed $(0.9$ and $5.4 \mathrm{~cm}$, respectively). The average values of SDIH $d^{\prime}$ calculated from the pure optical size changes, expansion or contraction $(9.3$ and $0.9 \mathrm{~cm}$, respectively) are less than the averages calculated from the mixed optical size changes, expansions or contractions (14.0 and $5.4 \mathrm{~cm}$, respectively). An ANOVA was completed on the computed values of SDIH $d^{\prime}$ using the factors of pure optical change versus mixed optical change and expansion versus contraction. Both factors were statistically significant $[F(1,11)=7.66, p<.02$, and $F(1,11)=6.08, p<.03$, respectively $]$.

\section{Discussion}

The obtained $d^{\prime}$. The amount of Obt. $d^{\prime}$ in Experiment 1 as indicated by the sagittal adjustments associated with the perceived motion in depth from the pure optical size changes and the perceived growth in depth from the mixed size optical change (a mixture of pure optical size change and the relative size cue of depth) are essentially the same. The overall average Obt. $d^{\prime}$ for the former is $19.6 \mathrm{~cm}$ and for the latter it is $19.0 \mathrm{~cm}$. Also, it should be noted that the magnitude of the average values of Obt. $d^{\prime}$ from the optical expansions (whether pure or mixed) are somewhat less than those obtained from the optical contractions (whether pure or mixed), with overall averages of 17.0 and $21.8 \mathrm{~cm}$, respectively. If the perceptions in the contractions and expansions had been equal to the physical events simulated, it would be expected that the Obt. $d^{\prime}$ would have been larger from the contractions than from the expansions. In the case of perfect simulations, the average perceived sagittal motion would have been 28.1 and $94.5 \mathrm{~cm}$ for Situations Ie and Ic, respectively. This expectation results from assuming a perceptual parallel to the inverse square law, in which, in physical space, a larger sagittal motion or depth will occur for the same change in a distance cue (e.g., in the present study the same change in visual angle) as the per- 
ceived distance of the configuration from the observer is greater (Gogel, 1964; Ono, Rivest, \& Ono, 1986; Rivest, Ono, \& Saida, 1989; Wallach \& Zuckerman, 1963). However, as was indicated by applying the obtained perceived sizes to calculate the perceived egocentric distances, $D^{\prime}$, from the single static stimulus presentations, using Equation 2, the optical contractions in Experiment 1 started at a perceived overall average of $9.6 \mathrm{~cm}$ closer than the optical expansions. This should have reduced but probably would not have eliminated the Obt. $d^{\prime}$ differences that would be expected to occur between the simulated optical contractions and expansions. Another possible contribution to the reduction in the expected Obt. $d$ ' between expansion and contraction is the "far anchor effect" described by Mershon et al. (1993). It was found that, if a stereoscopic depth between two targets with no salient egocentric cues to distance was changed, the perceptually nearer, more frequently than the perceptually farther, target appeared to be displaced in distance regardless of which target was changed in convergence. Assume that the far anchor effect applies to the monocularly viewed stimuli of the present study. In that case, following one or several presentations of simulated depth motion, it might be expected that the beginning and ending terminal stimuli of the optical contraction would be perceived as being displaced in depth toward the ending and beginning stimuli, respectively, of the optical expansion. As a consequence, the difference in the $d^{\prime}$ obtained between these two dynamic conditions would be further reduced or removed.

Relation between $\boldsymbol{S}_{\mathrm{S}}^{\prime} / \boldsymbol{S}_{\mathrm{L}}^{\prime}$ and SDIH $\boldsymbol{d}^{\prime}$. Since Equation 2 uses the distributions of $S_{\mathrm{S}}^{\prime}$ and $S_{\mathrm{L}}^{\prime}$ obtained in the experiment to calculate the distributions of SDIH $d^{\prime}$, a positive relation is present between the magnitude of size constancy $S_{\mathrm{S}}^{\prime} / S_{\mathrm{L}}^{\prime}$ and the magnitude of the calculated value of SDIH $d^{\prime}$. Thus, the values of $S_{\mathrm{S}}^{\prime} / S_{\mathrm{L}}^{\prime}$ calculated from Table 1 and the values of SDIH $d^{\prime}$ calculated from Equation 2 and shown in Table 1 increase together in magnitude as obtained from the same series of optical changes. As noted previously from Situations Ic, IIc, Ie, and IIe for $S_{\mathrm{S}}^{\prime} / S_{\mathrm{L}}^{\prime}$, these are in the order of $0.312,0.338$, 0.365 , and 0.403 . For SDIH $d^{\prime}$ calculated from Equation 2, as expected these are in the same order: $0.9,5.4,9.3$, and $14.0 \mathrm{~cm}$, respectively. However, under the conditions of Experiment 1 the SDIH $d^{\prime}$ predicted in this manner (as shown in Table 1) usually is substantially less than the $d^{\prime}$ measured by the sagittal adjustments (the Obt. $d^{\prime}$ of Table 1). The difference between the Obt. $d^{\prime}$ and the SDIH $d^{\prime}$ of Table 1 indicates the amount of obtained $d^{\prime}$ that, according to Equation 2, is unsupported by the amount of size constancy available. This remainder $d^{\prime}$ is not only significantly greater for the contracting optical changes, Ic and IIc, than for the expanding optical changes, Ie and IIe, but is also significantly greater for the pure optical changes, Ie and Ic, than for the mixed optical changes, IIe and IIc. Even though the amount of optical size change (the change in visual angle) was the same in the four sit- uations, the amount of size constancy and thus the involvement of size constancy and the SDIH in predicting the sagitally perceived motion in depth and growth differed considerably in the different situations.

\section{EXPERIMENT 2}

In the mixed optical changes (Situations IIe and IIc) in Experiment 1, the observer viewed an increasing number of stimulus squares in the successive presentations of a single sequence, thereby producing a gradient of relative size (perspective) that appeared to grow in depth either toward the observer (Situation Ile) or away from the observer (Situation IIc). It was found that the amount of Obt. $d^{\prime}$ that could be explained by the SDIH using the obtained values of $S_{\mathrm{S}}^{\prime}$ and $S_{\mathrm{L}}^{\prime}$ (the SDIH $d^{\prime}$ of Table 1) was greater in the mixed optical changes than in the pure optical changes even though the magnitude of the Obt. $d^{\prime}$ was essentially the same in both. This result could be attributed to the greater involvement of the relative size or perspective cue that occurred in the mixed as compared with the pure optical change. It follows that still more of the Obt. $d^{\prime}$ possibly might be explained by Equation 2 if the relative size (perspective) cue were continuously visible throughout the series of presentations. This condition, in which the full amount of optical size change is present in each presentation of the sequence, is illustrated in Situation III of Figure 1. It is called the pure relative size or perspective cue situation and it contains the same amount of optical size differences (optical size change) between the stimuli of different physical sizes as was present in Situations I and II of Experiment 1. Only the mixed situations of Experiment 1 and the added Situation III were used in Experiment 2.

\section{Method}

The apparatus, monocular viewing, stimulus, instructions, procedures, measures obtained, and 11 of the 12 observers were identical to those used in Experiment 1. However, in Experiment 2, which consisted of the presentation of Situations II and III, Situation III of Figure 1 (called a pure relative size or pure perspective cue) was presented instead of Situations Ie and Ic of Experiment 1. The successive stimulus presentations of Situation III (each presentation of which, as shown in Figure 1, contained all seven of the stimulus sizes of Experiment 1) were identical to each other and used, where appropriate, the presentation time intervals described in Experiment I. In the presentations of Situation III, of course, there was no distinction between expansion and contraction. However, to make the procedure in presenting Situation III comparable to the procedure in presenting Situations IIe and IIc, Situation III was presented twice, and a single (static) square stimulus with a size of either 22.2 or $6.6 \mathrm{~cm}$ was presented equally often before each presentation of Situation III. This resulted in measures of obtained $S^{\prime}$ and obtained $d^{\prime}$ in Situation III equaling the number of these measures obtained in the mixed optical situations of this and the previous experiment.

\section{Results}

The average results from Experiment 2 are shown in Table 2, which has the same structure as Table 1 . Since 
Table 2

Average Results From Experiment 2

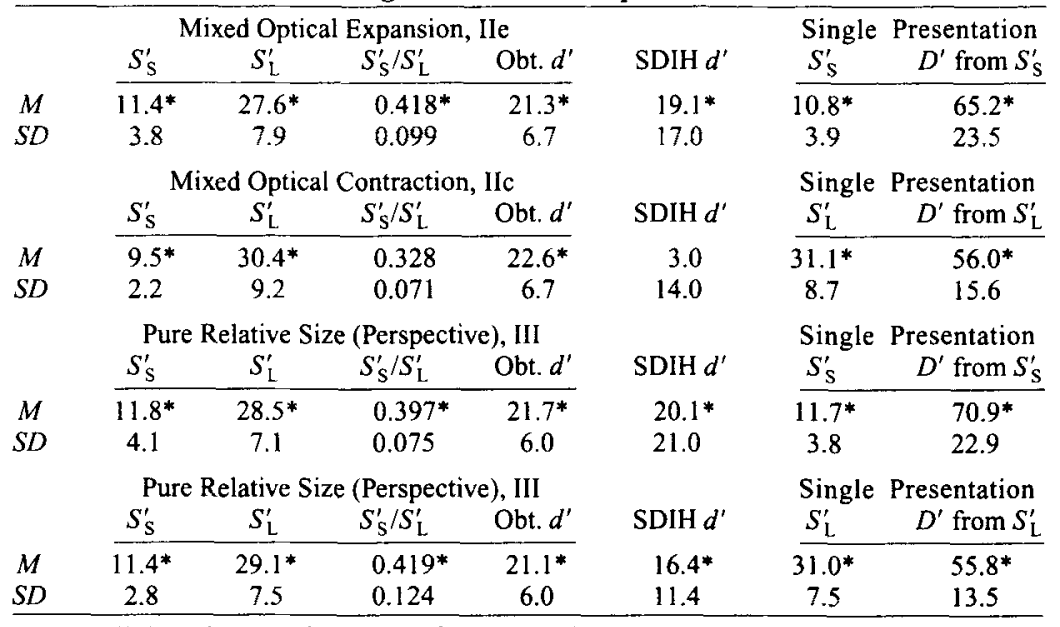

Note-All the values of $S^{\prime}, d^{\prime}$, and $D^{\prime}$ are in centimeters. The values of $S_{\mathrm{S}}^{\prime} / S_{\mathrm{L}}^{\prime}$ are the averages of the ratios of the distributions of $S_{\mathrm{S}}^{\prime} / S_{\mathrm{L}}^{\prime}$, not the ratio of the average values of $S_{\mathrm{S}}^{\prime}$ and $S_{\mathrm{L}}^{\prime}$ shown in the previous two columns. The statistically significant differences are from zero for all of the means except those labeled $S_{\mathrm{S}}^{\prime} / S_{\mathrm{L}}^{\prime}$, in which the significant differences are from zero constancy, where zero constancy is $2 \tan \left(\theta_{S} / 2\right) / 2 \tan \left(\theta_{L} / 2\right)$, or 0.297 in the present study. The values of perceived egocentric distance $\left(D^{\prime}\right)$ for the single (static) presentations were computed using Equation $1 .{ }^{*} p<.01$.

in Experiment 2 expansion and contraction were variables only in Situation II, the results from Situations II and III sometimes were analyzed separately.

Perception of size. The left two columns of Table 2 show the average left-to-right extent of the perceived linear sizes, $S_{\mathrm{S}}^{\prime}$ and $S_{\mathrm{L}}^{\prime}$, of the smallest $(6.6 \mathrm{~cm}$ wide) and largest ( $22.2 \mathrm{~cm}$ wide) stimuli used. Again, the third column gives the average $S_{\mathrm{S}}^{\prime} / S_{\mathrm{L}}^{\prime}$ as determined from the distribution of $S_{\mathrm{S}}^{\prime} / S_{\mathrm{L}}^{\prime}$ ratios. If zero or perfect size constancy had occurred, the ratio $S_{\mathrm{S}}^{\prime} / S_{\mathrm{L}}^{\prime}$, as in Experiment 1, would have been 0.297 and 1.00 , respectively. Clearly, again, only a limited amount of size constancy was present. The average $S_{\mathrm{S}}^{\prime} / S_{\mathrm{L}}^{\prime}$ in Table 2 is 0.418 for Situation IIe and 0.328 for Situation IIc. For the two presentation series of Situation III, the two averages of $S_{\mathrm{S}}^{\prime} / S_{\mathrm{L}}^{\prime}$ were 0.397 and 0.419. As in Experiment 1, the amount of size constancy from the mixed optical expansion (IIe) is greater than that from the mixed optical contraction (IIc), whereas the amounts of size constancy obtained in the mixed optical expansion and in the pure relative size cue (III) are quite similar.

A two-factor ANOVA was completed on the average $S_{\mathrm{S}}^{\prime}$ and $S_{\mathrm{L}}^{\prime}$ data of Situations IIe and IIc in the left two columns of Table 2 for the factors of expansion versus contraction and small versus large stimulus. Only the latter factor was statistically significant at, at least, the .05 level $[F(1,11)=125.95, p<.01]$. The interaction between the two factors that would have indicated a difference in the size constancy obtained from the expansion and contraction was in the direction expected from Experiment 1 , but it did not achieve the .05 level with a two-tailed test $[F(1,11)=3.57, p=.09]$. An ANOVA of the $S_{\mathrm{S}}^{\prime} / S_{\mathrm{L}}^{\prime}$ using the results obtained from Situations IIe, IIc, and the averages of the two presentations series of Situation III also was completed. The differences among Situations IIc, IIe, and III were statistically significant $[F(2,22)=7.069$, $p<.01]$, with the results from IIc significantly less than the results from IIe or III at $p<.01$ and .02 , respectively, using the Scheffé comparison test. Thus, the average obtained ratio, $S_{\mathrm{S}}^{\prime} / S_{\mathrm{L}}^{\prime}$, indicating the amount of size constancy was greater (closer to 1.00) in Situations IIe and III than in Situation IIc, with the results from Situations IIe and III not significantly different.

A $t$ test was completed on the $S^{\prime}$ data of Situation III averaged over the two presentations of this situation. As expected, the difference between the perceived linear sizes of the large and the small stimuli was statistically significant $[t(11)=11.10, p<.01]$.

The overall results from Experiment 2 for the large and the small stimuli in the single (static) presentations shown in the two right columns of Table 2 differed in perceived linear size $\left(S^{\prime}\right)$, with this difference being statistically significant $[t(11)=9.95, p<.01]$. The values of perceived egocentric distance $\left(D^{\prime}\right)$ calculated from the difference between the largest $S_{\mathrm{L}}^{\prime}$ and smallest $S_{\mathrm{S}}^{\prime}$, in the single (static) conditions, averaged over Situations II and III using Equation 2, although not statistically significant using a two-tailed result $[t(11)=2.11, p=.06]$, were in the direction indicating that the perceived starting distance of the largest stimulus probably was closer to the observer than the perceived starting distance of the smallest stimulus (a difference of $12.1 \mathrm{~cm}$, i.e., $68.0-55.9 \mathrm{~cm}$ ). Also as in Experiment 1, according to the $D^{\prime}$ calculations, the perceived egocentric distance of both the small and 
the large stimuli in the single static presentations exceeded their physical distance at $40 \mathrm{~cm}$, as expected from the SDT.

Perception of depth. The average obtained values of $d^{\prime}$ from the sagittal adjustment of the posts (Obt. $\left.d^{\prime}\right)$ and the $d^{\prime}$ as calculated from $S_{\mathrm{S}}^{\prime}$ and $S_{\mathrm{L}}^{\prime}$ using Equation 2 (the SDIH $d^{\prime}$ ) is shown in the two center columns of Table 2 . The pattern of these results from the mixed optical changes is similar to that from Experiment 1, with Situations IIc and IIe having similar values of Obt. $d^{\prime}$ in the two experiments and with the SDIH $d^{\prime}$ in Situation IIc being much smaller than that in Situation IIe.

A $t$ test was completed on the Obt. $d^{\prime}$ of Table 2 for the factor of expansion versus contraction in Situation II. This factor was not statistically significant at the .05 level. An ANOVA using the factors of expansion versus contraction and Obt. $d^{\prime}$ versus SDIH $d^{\prime}$ was completed for Situation II of Experiment 2. The effect of both factors and their interaction were statistically significant $[F(1,11)=$ $9.27, p<.01, F(1,11)=9.78, p<.01$, and $F(1,11)=7.63$, $p<.05$, respectively]. A $t$ test of the Obt. $d^{\prime}$ versus SDIH $d^{\prime}$ applied to the results averaged over the two presentations of Situation III was not significant at the .05 level $[t(11)=$ $0.85, p>.05]$. It is concluded that the Obt. $d^{\prime}-\mathrm{SDIH} d^{\prime}$ obtained in Situations IIe and III in Table 2 were similar but differed from the Obt. $d^{\prime}-\mathrm{SDIH} d^{\prime}$ from Situation IIc. Also, a $t$ test was conducted for the effect of expansion versus contraction on the SDIH $d^{\prime}$ calculated in Situation II. This factor was found to be statistically significant $[t(11)=2.98, p<.01]$, again, consistent with the effect on the SDIH $d^{\prime}$ found in Situation II of Experiment 1.

\section{Discussion}

The pure relative size cue sequences (Situation III) of Experiment 2 produced no substantial increase in the ability of Equation 2, using the $S_{\mathrm{S}}^{\prime}$ and $S_{\mathrm{L}}^{\prime}$ values, to predict the Obt. $d^{\prime}$ beyond that demonstrated in Situation IIe of either Experiment 1 or 2. However, it will be noted in Situation IIe in both experiments and in Situation III of Experiment 2 that the $d^{\prime}$ obtained using the sagittal adjustments of the posts (Obt. $d^{\prime}$ ) was not much larger than that predicted from the SDIH (SDIH $d^{\prime}$ ) using Equation 2. Also, in Experiment 2, as in Experiment 1, the difference between the Obt. $d^{\prime}$ and the SDIH $d^{\prime}$ in Situation IIc remained substantial. Thus, Experiment 2 supports results obtained in Experiment 1 and in addition supports the ability of the SDIH (Equation 2) to predict the perceived depth (Obt. $\left.d^{\prime}\right)$ in Situation III as well as IIe and the failure of Equation 2 to even approximately predict the Obt. $d^{\prime}$ from Situation Ic in Experiment 1 and from Situation IIc in either experiment.

\section{GENERAL DISCUSSION}

\section{Factors Possibly Modifying the Obt. $d^{\prime}$}

It was noted that the perceived sagittal motion or displacement (Obt. $\left.d^{\prime}\right)$ in this study was considerably less than that expected from the motion simulated by the ex- pansion $(28.1 \mathrm{~cm})$ and contraction $(94.5 \mathrm{~cm})$, assuming the presence of perfect size constancy. This result has been attributed to (1) the clear lack of large amounts of size constancy, (2) possibly the far anchor effect described by Mershon et al. (1993), (3) residual cues indicating the constant distance of the stimuli, and (4) probable differences in the starting perceived distances of the expansions and contractions. A possible additional factor might be measurement errors in either the perceived linear sizes of the terminal stimuli or in their perceived sagittal separations or displacements. An error in the latter measure might result from an inability of the observers to sufficiently separate the posts in sagittal distance using the two hands because of a limitation in the observer's reach in adjusting the farther (movable) post. A somewhat conservative estimate of the largest distance to which the observers were able to reach in order to adjust the movable post in distance from the eyes under the conditions of the position of the head in the chinrest and the location of the posts below the alley floor is $50 \mathrm{~cm}$. With the stationary post $3 \mathrm{~cm}$ forward of the observer's eye, this permitted a sagittal measure at the largest reach of $50-3=$ $47 \mathrm{~cm}$. This was adequate, however, since the largest average Obt. $d^{\prime}$ was $22.6 \mathrm{~cm}$, as shown in Table 1, Situation Ic, and the largest sagittal separation of the posts provided by any observer in the entire study was $35.2 \mathrm{~cm}$. Similarly, the $d^{\prime}$ limitations from the SDIH found in the present study also cannot be attributed to adjustment restrictions in the measurement of perceived linear size. The largest average $S^{\prime}$ measure in the tables is $31.1 \mathrm{~cm}$, and the largest adjustment from any observer was $41.8 \mathrm{~cm}$, whereas, as indicated, the largest adjustment that could be obtained physically from the lateral adjustment apparatus was $47.3 \mathrm{~cm}$.

A study by Gogel and Eby (Experiment 1, 1997) confirms the substantial differences between the simulated and sagitally measured $d^{\prime}$ (i.e., Obt. $d^{\prime}$ ) under circumstances in which whole cycles starting with either an expansion or a contraction were used. In that experiment terminal stimulus sizes of 7.0 and $23.3 \mathrm{~cm}$ using continuous optical change were presented at $40 \mathrm{~cm}$, thereby providing simulations of 28.0 and $94.5 \mathrm{~cm}$, depending upon whether the beginning half-cycle was an optical expansion or contraction, respectively. The average obtained $d$ 's were 23.2 and $24.8 \mathrm{~cm}$ for the optical changes starting as expansions and contractions, respectively. These values, although somewhat larger than the 17.1 and $22.2 \mathrm{~cm}$ from Ie and Ic in Table 1 of the present study, are not greatly larger. In the Gogel and Eby study the same sagittal measuring apparatus was used as in the present study except that the near stationary post was $15.7 \mathrm{~cm}$ forward of the observer's eye, limiting the likely reaching range of sagittal measurement from the hand (post) separations to $50-15.7 \mathrm{~cm}$, or $34.3 \mathrm{~cm}$. The largest sagittal measure obtained from any observer, however, was $28.3 \mathrm{~cm}$, which was clearly within the possible reaching interval. It seems that no identifiable measurement artifact was present to limit the measures of $d^{\prime}$ obtained in 


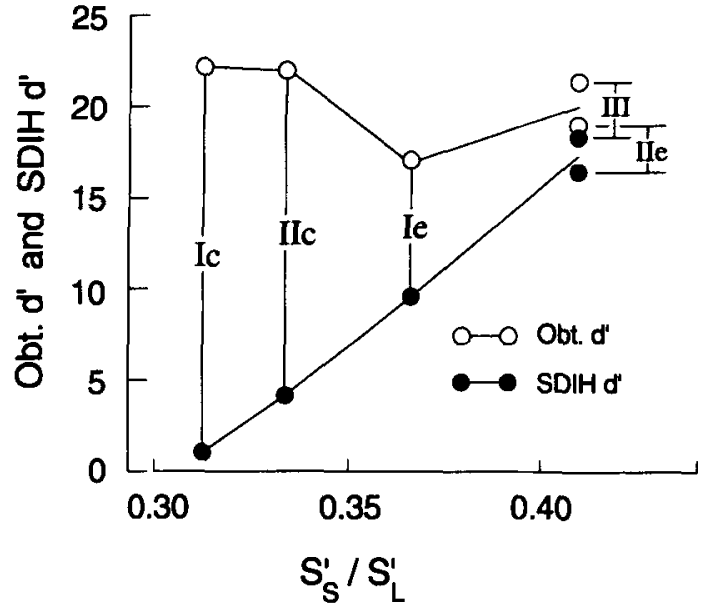

Figure 2. The ordinate values of the open circles are the perceived sagittal motions in depth (Obt. $d^{\prime}$ ) from the series of presentations obtained by the observer's adjustments of the sagittal distances between the posts. The ordinate values of the filled circles are the perceived motions in depth (size-distance invariance hypothesis [SDIH] $d^{\prime}$ ) as calculated from the perceived linear sizes of the terminal stimuli of the presentation series using Equation 2. The abscissa indicates the values of the $S_{\mathrm{S}}^{\prime} / S_{\mathrm{L}}^{\prime}$ measures of size constancy associated with each of the five situations of optical size change (Situations Ie, Ic, IIe, IIc, and III). The length of the vertical line joining the open and filled circles for each situation indicates the difference between the two sources of $d^{\prime}$ (i.e., Obt. $d^{\prime}-\operatorname{SDIH} d^{\prime}$ ). This is the amount of $d^{\prime}$ obtained that cannot be explained by the available amount of size constancy using the SDIH.

either of these studies and that, in the present study, neither the lack of continuous optical changes nor the use of half-cycles greatly reduced the magnitude of $d^{\prime}$ results obtained from the optical expansions and contractions. It is possible, of course, in both studies that the procedure of using open-loop hand (post) adjustments to measure the perception of sagittal depth or displacement underestimated the visual magnitude of the perceived depth or displacement due to some intrinsic error in relating visual and kinesthetic depth. Indeed, there is some evidence for this possibility, as indicated by the data of Figure 7 in Gogel and Tietz (1992b). However, even if this limitation had occurred in the present study, it would not invalidate the conclusion that the SDIH was not able to always predict the full Obt. $d$ '. Nor would it avoid the need to postulate an additional variable in order to explain the remaining $d^{\prime}$ (i.e., Obt. $d^{\prime}-\mathrm{SDIH} d^{\prime}$ ) that often occurred. However, kinesthetic underestimation was not likely to have occurred in the measurements of perceived linear size $\left(S^{\prime}\right)$ in the present study. In a study by Gogel, Wist, and Harker (1963), stimuli of different linear sizes were presented at a constant physical distance under multicue conditions and were measured with an openloop kinesthetic procedure. The result was that the measures using the separation of the hands were very similar to the physical linear sizes of the stimuli.

\section{Interrelations of $S_{\mathrm{S}}^{\prime} / S_{\mathrm{L}}^{\prime}$, SDIH $d^{\prime}$, and Obt. $d^{\prime}$}

Figure 2 provides a summary of the relation between perceived size constancy expressed by $S_{\mathrm{S}}^{\prime} / S_{\mathrm{L}}^{\prime}$, the SDIH $d^{\prime}$ predicted from Equation 2 using the values of $S_{\mathrm{S}}^{\prime}$ and $S_{\mathrm{L}}^{\prime}$, and the $d^{\prime}$ obtained from the sagittal adjustments of the posts (Obt. $\left.d^{\prime}\right)$ for the different situations used in the study. The data from both Experiments 1 and 2 are included and are averaged over the identical stimulus situations whether found in Experiment 1 or 2 . Thus, the data from Situation Ile are averaged over the two experiments, as are the data from Situation IIc, and the data from Situation III are averaged over the two presentations of Situation III in Experiment 2. The vertical distance between the two curves of Figure 2 indicates the difference in centimeters between the Obt. $d^{\prime}$ and the computed SDIH $d^{\prime}$ using Equation 2 for the various amounts of perceived size constancy expressed as $S_{\mathrm{S}}^{\prime} / S_{\mathrm{L}}^{\prime}$. The $d^{\prime}$ predicted from the SDIH, using Equation 2 (i.e., the SDIH $d^{\prime}$ ), is larger for the expansion than for the corresponding contraction in both the pure and the mixed optical changes. Also, the Obt. $d^{\prime}$ is more adequately predicted by the SDIH $d^{\prime}$ in the mixed optical size change than it is in the corresponding situations of pure optical size change. Furthermore, in both the mixed optical expansion (Situation IIe) and the pure relative size cue (Situation III), the amount of the Obt. $d^{\prime}$ that is predicted by the SDIH is almost complete. Clearly, from Figure 2, the ability of the measured amount of size constancy expressed through Equation 2 to predict the $d^{\prime}$ using the sagittal adjustments of the posts (the Obt. $d^{\prime}$ ) increased as the amount of size constancy increased until, in the case of Situations IIe and III, the amount of size constancy and thus the SDIH $d^{\prime}$ was almost completely appropriate to the amount of perceived motion or displacement in depth.

There are three quite general results from this study that need to be considered. One is why the amount of size constancy as measured by $S_{\mathrm{S}}^{\prime} / S_{\mathrm{L}}^{\prime}$ and therefore the amount of $d^{\prime}$ predicted from Equation 2 (SDIH $d^{\prime}$ ) was different in the different situations, as shown in Figure 2. Another is the identification of the factor or factors that produced the additional $d^{\prime}$ in Obt. $d^{\prime}$ not predicted from the SDIH using Equation 2. The third is why total $d^{\prime}$ (i.e., Obt. $d^{\prime}$ ) in Figure 2 did not vary directly with the component of Obt. $d^{\prime}$ predicted from the SDIH.

\section{Determinants of the $d^{\prime}$ Predicted From the SDIH}

The reason for the average differences in the amount of size constancy and thus the $d^{\prime}$ predictions from the SDIH using Equation 2 among Situations I, II, and III is found in the increased effectiveness of the distance cues of relative size (perspective) as the presentations changed from being completely successive in Situation I, to more simultaneous in Situation II, to completely simultaneous in Situation III. Generally, and as found here, visual information that is temporally simultaneous is perceptually more effective than the same information that is temporally separate. But, an effect also shown in Figure 2, 
for which there is no ready explanation, is the greater contribution of the SDIH to the Obt. $d^{\prime}$ that occurred in the optical expansions compared with the optical contractions in Situations I and II. However, it also should be noted that differences between optical expansions and contractions have been found previously in motion thresholds (Edwards \& Babcock, 1993) and, consistent with the differences in $S_{\mathrm{S}}^{\prime} / S_{\mathrm{L}}^{\prime}$ in the present study, in rigidity (Perrone, 1986).

\section{Primary and Secondary Processes}

An identification of the factor producing values of Obt. $d^{\prime}$ beyond those produced by the SDIH is found in a distinction between primary and secondary processes as discussed by Gogel $(1990,1993)$, Gogel and Eby (1997), Gogel and Da Silva (1987b), and Swanston and Gogel (1986). A general criterion of primary processes is specified in what is termed a theory of phenomenal geometry (Gogel, 1990). It is assumed that the primary process is always perceptual. The basic variables of the theory are (1) perceived direction, (2) perceived egocentric distance or perceived depth, and (3) the observer's perception of his/her own position or motion. From these basic variables, the derived perceptual variables of size, shape, orientation, and motion of the stimulus are obtained (Gogel, 1990, 1993; Gogel \& Tietz, 1992a, 1992b). Of particular interest for the present study, the perceived visual angle $\theta^{\prime}$, at least as an approximation, can be substituted for the physical visual angle in Equation 2 (Foley, $1965,1972,1991$ ), and, according to the theory of phenomenal geometry, the SDIH fits the criterion of being a primary process. This criterion of a primary process is that the perceptual variables associated with stimulus configurations are internally (intrinsically) consistent. However, it is clear that many instances occur in the experimental literature in which the SDIH as expressed by Equation 2 has been disconfirmed (see Foley, 1968). For example, perceived egocentric distance has been found using binocular observation and otherwise reduced conditions to be underestimated relative to a lateral perceived size. Perceptually equating a lateral size to an egocentric distance under these conditions results in a visual angle that is judged as less than the $45^{\circ}$ expected from the ratio of the perceived extents (Foley, 1972). In this case the variables of $S^{\prime}, D^{\prime}$, and $\theta^{\prime}$ or $\theta$ are not all consistent intrinsically when expressed in Euclidean geometry. An inconsistency in the basic variables of $S^{\prime}, D^{\prime}$, and $\theta$ or presumably $\theta^{\prime}$ is indicated in Figure 2 of the present study in those situations (Ic, IIc, and Ie) in which an appreciable amount of difference between Obt. $d^{\prime}$ and SDIH $d^{\prime}$ was obtained. Only in the case of Situations IIe and III, in which cues of changing perceived distance are increasingly effective (as is indicated by the increased amount of size constancy), was the primary process represented by the SDIH capable of predicting almost all of the $d^{\prime}$ obtained from the sagittal adjustments. Thus, to explain both the contribution of different amounts of size constancy to the Obt. $d^{\prime}$ and the limitations of the size con- stancy to fully account for the Obt. $d^{\prime}$ shown in Figure 2, it is hypothesized, consistent with the study by Gogel and Eby (1997) and Gogel and Da Silva (1987b), that two processes are involved in the simulation of optical expansions or contractions. One, the primary process, is entirely perceptual and results in internal consistency of the perceptual variables. The other, the secondary process, is cognitive and, if it were able to modify any of the basic perceptual variables, would result in inconsistencies among the perceptual variables without appropriately modifying the remaining perceptual variables. Sometimes both processes contribute substantially to the perception of sagittal motion. In the present study, the primary process is dominant in Situations IIe and III; the secondary process is almost completely dominant in Situation Ic, and dominant but less so in Situation IIc, and both processes contribute approximately equally in Situation Ie. Unlike the primary process, which, in the case of the optical changes, is identified with the SDIH, the secondary (cognitive) process, as found in the present study and in the previous studies by Gogel and Eby (1997) and by Swanston and Gogel (1986), as will be discussed, is a consequence of off-sized perceptions.

\section{Secondary Processes and Off-Sized Perceptions}

The secondary process needed in an explanation of the differences between Obt. $d^{\prime}$ and SDIH $d^{\prime}$, as shown in Figure 2, is hypothesized to be the result of off-sized perceptions. Examples of off-sized perceptions or off-sized judgments are readily found when familiar objects are viewed from a large distance. For instance, houses viewed from an airplane at a considerable height look much smaller than normal. This happens because, in the absence of effective distance cues at the far distance, the houses appear closer than they are physically $\left(D^{\prime}<\mathrm{D}\right)$ and in agreement with the SDIH (Equation 1), they also appear smaller $\left(S^{\prime}\right)$ than would be expected from their familiar size $\left(S_{\mathrm{c}}\right)$. However, the information that the houses are perceived to be smaller than normal $\left(S^{\prime}<S_{\mathrm{c}}\right)$ is information indicating to the observer that they are at a greater distance $\left(D_{\mathrm{c}}\right)$ than the distance $\left(D^{\prime}\right)$ at which they are perceived. This result can be expressed generally as

$$
D_{\mathrm{c}}=D^{\prime}\left(S_{\mathrm{c}} / S^{\prime}\right),
$$

where $D_{\mathrm{c}}$ is the observer's verbal report of the distance of the stimulus, $S_{\mathrm{c}}$ is the familiar size of the stimulus, and $S_{\mathrm{c}} / S^{\prime}$ is the inverse of the off-sized judgment.2 It will be noted from this description that the response $D_{\mathrm{c}}$ when it differs from the perceived egocentric distance $D^{\prime}$ tends to correct the error in the perceived egocentric distance, thus making the judgment of egocentric distance of the stimulus more veridical. Also, according to Equation 3 the response $D_{c}$ from off-sized perceptions cannot occur in the complete absence of a primary process, as is reflected in the presence of the variables $S^{\prime}$ and $D^{\prime}$ in Equation 3.

A more general description of off-sized judgments or off-sized perceptions includes cases in which the presentation of a geometrical object (an object without a fa- 
miliar size) having the same shape as a smaller or larger stimulus presented subsequently becomes the standard size for determining the off-sized characteristic of the subsequent stimulus. Likely examples of this are the sizedistance paradox and the moon illusion, in which the tendency of the observer in these instances is to report the off-sized object as being at a greater or lesser distance, respectively, than the distance at which it appears (see Gogel \& Mertz, 1989).

The question of whether familiar size is a substantial determiner of perceived egocentric distance has considerable theoretical importance because (1) it provides the possibility of specifying perceived distances that are far from the observer, (2) it is frequently present, and (3) since it obviously is the result of experience, it represents the possibility of cognitive processes intruding into perceptual processes. But, strong evidence is available from a series of studies to support the conclusion that the familiar size of a stimulus object, although capable of clearly modifying the reported distance of the object, does not contribute substantially to the perceived distance of the object (Gogel, 1969, 1976, 1981; Gogel \& Da Silva, 1987a, 1987b; Gogel \& Newton, 1969; Predebon, 1987, 1990, 1991, 1992a, 1992b, 1993, 1994; Predebon \& Wolley, 1994). However, as noted, a plain (geometrical) object such as an outline square (an object without any indication of a familiar size) that is perceived to increase or decrease in size as the visual angle of the object increases or decreases (a dynamic condition) also can provide offsized perceptions of the object as compared with a preceding presentation or preceding series of presentations of the object. There is evidence to suggest in this dynamic case, however, that, unlike the static case, in which only a single object is presented, the stimulus will be perceived substantially as moving or displaced in depth, with the direction of motion or displacement toward or away from the observer depending upon whether the offsized judgments are increasing or decreasing in size, respectively (Gogel \& Eby, 1997; Swanston \& Gogel, 1986). This evidence for the ability of dynamic changes in the off-sized perception to modify perceived, not simply reported, distance was obtained in the study by Swanston and Gogel (1986) by measuring the illusory lateral motion of the stimulus concomitant with a lateral motion of the head in a situation in which Equation 2 was not able to predict any of the substantial $d^{\prime}$ being measured. This, then, is an instance of a cognitive effect (from successive, sequential, changes in off-sized perceptions) modifying the perception of sagittal motion. In this case, the presence of secondary (cognitive) processes of offsized perceptions can modify substantially the observer's perception of sagittal motion or sagittal distance even if size constancy is totally absent. Thus, in Situation Ic in Experiment 1, in which size constancy was essentially absent, the average Obt. $d^{\prime}$ (among the largest measured in the study) was very likely entirely the result of off-sized perceptions. It is as though the observer often assumes that an object of constant shape but changing in perceived linear size proportional to its changes in visual angle is actually the same object, and, under dynamic circumstances this constant identity can produce a perception of motion in depth despite a lack of appropriate amounts of size constancy.

The combination of effects from dynamic $S_{\mathrm{c}} / S^{\prime}$ and SDIH. It is hypothesized in the present study and the study of Gogel and Eby (1997) that the perceived sagittal depth or displacement from geometrical off-sized stimuli and from the amount of size constancy $\left(S_{\mathrm{S}}^{\prime} / S_{\mathrm{L}}^{\prime}\right)$ present will combine in producing the total perception Obt. $d^{\prime}$. Rather than perceiving a $d^{\prime}$ from each of these two sources separately, they are effective in combination. But, this is not an unusual result for perceptions of extent that occur in either a frontoparallel or sagittal plane. For example, if accommodation that by itself produces a perceived distance of $1.0 \mathrm{ft}$ is combined with convergence that by itself would produce a perception of distance of $3.0 \mathrm{ft}$, the two together can result in the single perception of the stimulus being at, perhaps, $2.0 \mathrm{ft}$. Or, consider the perception of tilt that occurs when a vertically moving stimulus point is surrounded by a horizontally moving frame. The combination of the perceived vertical motion as determined from the physical motion of the point and its perceived horizontal motion induced by the moving frame produces the single perception of tilt in the motion of the stimulus point. It is not unusual for multiple sources of motion or displacement to combine in a final single resultant.

The combined contribution of secondary process $S_{\mathrm{c}} / S^{\prime}$ from Equation 3 and the primary process from the degree of size constancy provides an answer to the problem of why Obt. $d^{\prime}$ in Figure 2 between the optical expansions Ie and IIe or between the optical contractions Ic and IIc are not more different. The total Obt. $d^{\prime}$ in each comparison is determined by that contributed by each of the two sources. But the magnitude of the changes in off-sized perceptions by definition is less as the amount of linear size constancy is greater. The result is that the combined contribution to Obt. $d^{\prime}$ for the same direction and magnitude of optical change in Situations I and II tends to remain approximately the same.

Implications and relations to other studies. The amount of perceived size constancy, defined as the ratio of the perceived linear sizes $\left(S_{\mathrm{S}}^{\prime} / S_{\mathrm{L}}^{\prime}\right)$ of the stimuli at the terminal distances, differed for the different stimulus conditions as shown in Figure 2 and was considerably less than complete in all the situations; that is, the stimulus object in all the situations clearly appeared to grow larger or smaller as it appeared to move toward or away from the observer, respectively. This result is in general agreement with results obtained using pure optical expansions and contractions (Gogel \& Eby, 1997; Swanston \& Gogel, 1986), but it is not in agreement with a number of other studies (Hershenson, 1992a, 1992b, 1993; see also Johansson, 1977), in which it was concluded that the changing visual angles of constant shape, under quite reduced cues of changing distance, resulted in perceptions of object rigidity (complete size constancy). Although there are 
apparatus and procedural differences among these various studies, two concerns are important regarding the size responses obtained in such experiments. One concern from the point of view supported by the present study is whether the perceptions of the unchanging shape of the stimuli produced the responses of constant physical size rather than the responses reflecting the linear sizes of the stimuli as actually perceived. In the studies by Gogel and Eby (1997) and by Swanston and Gogel (1986), there is a somewhat parallel but different concern. In those studies the question can be raised whether the responses indicating an amount of linear size constancy much less than perfect are, at least partially, responses to the changing visual angle of the stimulus rather than responses to the perceived linear size of the stimulus. Swanston and Gogel discussed reasons for rejecting the latter possibility in their study. In the study by Gogel and Eby, an instructional procedure for avoiding confusion between the responses to perceived linear size and perceived angular size was introduced. It was concluded in both studies that the $S^{\prime}$ responses were indeed responses to perceived linear size rather than to perceived (or physical) angular size.

In the study by Swanston and Gogel (1986, see Experiment 1) full (complete) cycles of optical change starting with an optical expansion were presented. In the Gogel and Eby (1997) study full cycles starting with either an optical expansion or an optical contraction were used. An unexplained difference between these two studies was the finding in the former study that, under otherwise reduced conditions, the SDIH predicted none of the total motion perceived. In the latter study the SDIH using Equation 2 predicted about half the total motion perceived, with the average $d^{\prime}$ predicted from Equation 2 somewhat less when the starting optical change was a contraction rather than an expansion. In the present study, only halfcycles of either seven successive expansions or contractions were presented repetitively. When only an expansion was presented (Situation Ie), the calculated contribution of the SDIH was about $50 \%$ of the total perceived sagittal motion. When only a contraction was presented, the prediction from the SDIH, using Equation 2 and the small amount of size constancy present, was that essentially no perceived sagittal motion should have occurred. It seems from these comparisons that the prediction from the SDIH (using Equation 2) as to the total amount of perceived sagittal motion is different when only the expansion or contraction component alone is presented repetitively, compared with the repetitive presentation of complete cycles of optical change.

A more general expression for the SDIH is provided by substituting the term for perceived visual angle $\left(\theta^{\prime}\right)$ for the term for physical visual angle $(\theta)$ in Equations 1 and 2 (Gogel \& Da Silva, 1987a; McCready, 1965, 1985, 1986). In terms of these more general equations, Equations 1 and 2 , as used in the present study, would be accurate equations for the SDIH only if $\theta^{\prime}=\boldsymbol{\theta}$. However, substituting terms other than $\theta^{\prime}$ for $\theta$ has been proposed. According to these possibilities, $\theta^{\prime}$ equals $K \theta$ (see Gogel $\&$ Da Silva, 1987a), $K \theta^{\text {n }}$ (Foley, 1967, 1968; Gogel, 1971; Oyama, 1974), or $a \theta+b$ (Higashiyama \& Shimono, 1994), where $K, n, a$, and $b$ are constants whose values depend upon the conditions. In direct measurements of $\theta^{\prime}$ it has been found (often using quite different procedures) that the size of $\theta^{\prime}$ obtained can substantially exceed that of $\theta$ (Higashiyama, 1992), can equal $\theta$ (Gogel, 1982), or in some cases can be considerably less than $\theta$ (see Gogel \& Eby, 1997). The question therefore occurs as to whether the pattern of values of SDIH $d^{\prime}$ obtained from the different situations of the present study would have been unchanged had measured values of $\theta^{\prime}$ different from $\theta$ been used in Equation 2. It seems probable, however, that the pattern of these results, although not necessarily their measured values, would have remained essentially unchanged. The reason for this is that the optical size changes between the stimuli in all of the situations were the same. Thus the important determiner of the pattern of differences between the calculated SDIH $d^{\prime}$ from the different situations of the study is the different values of $S_{\mathrm{S}}^{\prime}$ and $S_{\mathrm{L}}^{\prime}$ and not the relation between $\theta$ and $\theta^{\prime}$, which could be expected to be similar in all of the situations. Indeed, there is some reason to conclude that the perception of visual angle is not readily modified in the context of satisfying the SDIH (Gogel \& Eby, 1997). Also, one aspect of the present data tends to support the assumption that $\theta_{\mathrm{S}} / \theta_{\mathrm{L}}$ equals $\theta_{\mathrm{S}}^{\prime} / \theta_{\mathrm{L}}^{\prime}$. It was noted previously that if $S_{\mathrm{S}}^{\prime} / S_{\mathrm{L}}^{\prime}$ equals $\tan \left(\theta_{\mathrm{S}} / 2\right) / \tan \left(\theta_{\mathrm{L}} / 2\right)$ in Equation 2 , the calculated SDIH $d^{\prime}$ according to Equation 2 should be zero. This is essentially what was found from the data of Situation Ic. In that case, $S_{\mathrm{S}}^{\prime} / S_{\mathrm{L}}^{\prime}=0.304, \tan \left(\theta_{\mathrm{S}} / 2\right) / \tan \left(\theta_{\mathrm{L}} / 2\right)=0.297$, and the calculated value of SDIH $d^{\prime}$ from Equation 2 was small $(0.9 \mathrm{~cm})$. Thus, since the changes in visual angle of the stimuli were the same in all the situations of the study, the fact that Equation 2 in Situation Ic fits the relation between $S_{\mathrm{S}}^{\prime} / S_{\mathrm{L}}^{\prime}$ and $\tan \left(\theta_{\mathrm{S}} / 2\right) / \tan \left(\theta_{\mathrm{L}} / 2\right)$ suggests that it is likely that Equation 2 is the appropriate equation for use throughout the study. In addition, it should be noted that the values of SDIH $d^{\prime}$ from Equation 2 were similar to the values of Obt. $d^{\prime}$ in Situations IIe and III. Since Situations IIe and III might be expected to be the situations of the study in which the predictions from Equation 2 would be most likely to apply, such results provide some confidence in the likelihood that Equation 2 adequately represents the apportionment process between changes in perceived linear size and perceived depth and also provides a valid representation of the SDIH.

A main contribution of the present study is that it indicates that it is premature to conclude from the inability of the SDIH to fully predict the perception of simulated sagittal motion in an optical expansion or contraction that the SDIH should be considered as irrelevant to that phenomenon. The involvement of the SDIH in such phenomena is necessary if for no other purpose than to provide the perception of size, $S^{\prime}$, and distance, $D^{\prime}$, necessary for the occurrence of off-sized perceptions, as shown in Equation 3 . In addition, the present study suggests that the 
SDIH is increasingly involved in the perception of sagittal motion or displacement as cues of changing perceived distance are increasingly effective. According to the present study the SDIH expressed by Equation 2 inhibited the contribution of off-sized perceptions to Obt. $d^{\prime}$ in Situations IIe and III long before the presence of off-sized judgments were completely eliminated.

\section{Summary}

The main results and interpretations from the present study are as follows. In none of the situations presented was perceived stimulus rigidity complete or even approximated (perfect size constancy clearly was not present). However, as shown in Figure 2, the amount of size constancy, defined by the ratio of the smallest to the largest perceived linear size of the terminal stimuli, increased from the situations of successive presentations (pure optical expansion or contraction) to that of simultaneous presentations (pure perspective cue). Associated with this increase was an increase in the ability of the SDIH, using Equation 2, to predict the perceived motion or displacement in depth (Obt. $\left.d^{\prime}\right)$. However, as shown in Figure 2, there was no systematic change in the perceived sagittal motion or sagittal displacement (Obt. $d^{\prime}$ ) between the different situations of the same type, that is, Situation I or Situation II, as the amount of linear size constancy (or of SDIH $d^{\prime}$ ) increased. This is explained by the perceived sagittal motion or displacement provided by off-sized perceptions necessarily being inversely related to the amount of linear size constancy present. Thus, in the dynamic conditions of the present study, offsized perceptions added a variable component of perceived motion in depth or displacement that tended to compensate for the errors in perceived depth or distance that occurred because of different amounts of incompleteness in the size constancy available.

Several results obtained in the present study, however, remain to be explained. It is reasonable to expect that the relative size cue will be more effective when the stimuli are presented simultaneously rather than successively. But it is not clear what heuristic factors, in the absence of an effective assumption of size equality, are controlling the amount of size constancy available in the different situations. It is likely that a similar problem is present in explaining the effect of the depth cue of relative motion parallax, in which magnitudes of lateral motion of the stimuli are substituted for the values of visual angular extent in the relative size or perspective cue of depth. This problem in the present study is accented by the smaller amount of size constancy (or contribution of the SDIH to the Obt. $d^{\prime}$ ) obtained in the pure or mixed contraction as compared with the pure or mixed expansion.

There is also a general issue to which the results from the present study together with the results from the study by Swanston and Gogel (1986) and Gogel and Eby (1997) are pertinent. When inconsistencies in the relation between response variables occur, it often is taken as an in- dication that the geometry of the perceptual world is non-Euclidean and that a different perceptual geometry is needed. A variety of non-Euclidean geometries have been applied in attempts to understand perceived visual space (see Foley, 1991; Wagner, 1985). However, it seems that no single geometry has yet been found that is consistent with perceived visual space under all the conditions tested, with Euclidean geometry becoming increasingly appropriate as the amount of visual spatial information available to the observer is increased and the stimuli are increasingly frontoparallel. A point of view alternative to applying a non-Euclidean geometry, in agreement with the present study and the cited two studies, is that Euclidean geometry is appropriate for the perceived as well as the physical visible world. It is suggested that intrinsic inconsistencies in the variables of the perceptual world, when they occur, are the result of the intrusion of cognitive processes that, although useful in reducing some of the inaccuracies or other limitations of the perception of the visual world, produce the inconsistencies. Probably, as considered by Gogel and Eby (1997), this occurs because the modification of one of the perceptions by the cognition fails to result in the change in other perceptions needed if the integrity of the geometry is to be maintained.

\section{REFERENCES}

EDwARDS, M., \& BABCOCK, D. R. (1993). Asymmetries in the sensitivity to motion in depth: A centripetal bias. Perception, 22, 1013-1023. FOLEY, J. M. (1965). Visual space: A scale of perceived relative direction. Proceedings of the Seventy-Third Annual Convention of the American Psychological Association, 1, 49-50.

FOLEY, J. M. (1967). Binocular disparity and perceived relative distance: An examination of two hypotheses. Vision Research, 7, 655-670

FOLEY, J. M. (1968). Depth, size and distance in stereoscopic vision. Journal of Experimental Psychology: Human Perception \& Performance, 11, 133-149.

FOLEY, J. M. (1972). The size-distance relation and intrinsic geometry of visual space: Implications for processing. Vision Research, 12, 323-332.

FOLEY, J. M. (1991). Binocular space perception. In D. M. Regan (Ed.), Vision and visual dysfunction: Binocular vision and psychophysics (Vol. 9, pp. 75-91). New York: Macmillan.

GoGEL, W. C. (1964). The perception of depth from binocular disparity. Journal of Experimental Psychology, 67, 379-386.

GoGEL, W. C. (1969). The effect of object familiarity on the perception of size and distance. Quarterly Journal of Experimental Psychology, 21, 239-247.

GoGEL, W. C. (1971). The validity of the size-distance invariance hypothesis with cue reduction. Perception \& Psychophysics, 9, 92-94.

GoGEL, W. C. (1976). An indirect method of measuring perceived distance from familiar size. Perception \& Psychophysics, 20, 419-429.

GOGEL, W. C. (1981). The role of suggested size in distance responses. Perception \& Psychophysics, 30, 149-155.

GOGEL, W. C. (1982). Analysis of the perception of motion concomitant with a lateral motion of the head. Perception \& Psychophysics, 32, $241-250$.

GOGEL, W. C. (1990). A theory of phenomenal geometry and its applications. Perception \& Psychophysics, 48, 105-123.

GoGEL, W. C. (1993). The analysis of perceived space. In S. C. Masin (Ed.), Foundations of perceptual theory (pp. 113-182). New York: Elsevier.

Gogei, W. C., \& DA Silva, J. A. (1987a). Familiar size and the theory of off-sized perceptions. Perception \& Psychophysics, 41, 318-328. 
Gogel, W. C., \& DA Silva, J. A. (1987b). A two-process theory of the response to size and distance. Perception \& Psychophysics, 41, 220-238.

GOGEL, W. C., \& EBY, D. W. (1997). Measures of perceived linear size, sagittal motion, and visual angle from optical expansions and contractions. Perception \& Psychophysics, 59, 783-806.

GogeL, W. C., \& MERTZ, D. L. (1989). The contribution of heuristic processes to the moon illusion. In M. Hershenson (Ed.), The moon illusion (pp. 235-258). Hillsdale, NJ: Erlbaum.

Gogel, W. C., \& Newton, R. E. (1969). Perception of off-sized objects. Perception \& Psychophysics, 5, 7-9.

Gogel, W. C., \& TiETZ, J. D. (1992a). Absence of compensation and reasoning-like processes in the perception of orientation in depth. Perception \& Psychophysics, 51, 309-318.

Gogel, W. C., \& TheTz, J. D. (1992b). Determinants of the perception of sagittal motion. Perception \& Psychophysics, 52, 75-96.

Gogel, W. C., Wist, E. R., \& HARKer, G. S. (1963). A test of the invariance of the ratio of perceived size to perceived distance. American Journal of Psychology, 76, 537-553.

HERSHENSON, M. (1992a). The perception of shrinking in apparent motion. Perception \& Psychophysics, 52, 671-675.

Hershenson, M. (1992b). Size-distance invariance: Kinetic invariance is different from static invariance. Perception \& Psychophysics, 51, 541-548.

HERSHENSON, M. (1993). Structural constraints: Further evidence from apparent motion in depth. Perception, 22, 323-334.

HigaShIYAMA, A. (1992). Anisotropic perception of visual angle: Implications for the horizontal-vertical illusion, overconstancy of size, and the moon illusion. Perception \& Psychophysics, 51, 218-230.

Higashiyama, A., \& Shimono, K. (1994). How accurate is size and distance perception for very far terrestrial objects? Function and causality. Perception \& Psychophysics, 55, 429-442.

JOHANSSON, G. (1977). Spatial constancy and motion in visual perception. In W. Epstein (Ed.), Stability and constancy in visual perception: Mechanisms and processes (pp. 375-419). New York: Wiley.

MCCREADY, D. (1965). Size-distance perception and accommodationconvergence micropsia-A critique. Vision Research, 5, 189-206.

MCCREADY, D. (1985). On size, distance, and visual angle perception. Perception \& Psychophysics, 37, 323-334.

McCready, D. (1986). Moon illusions redescribed. Perception \& Psychophysics, 39, 64-72.

Mershon, D. H., Jones, T. A., \& TAYlor, M. E. (1993). Organizational factors and the perception of motion in depth. Perception \& Psychophysics, 54, 240-249.

ONo, M. E., RIVEST, J., \& ONo, H. (1986). Depth perception as a function of motion parallax and absolute-distance information. Journal of Experimental Psychology: Human Perception \& Performance, 12, 331-337.

OYAMA, T. (1974). Perceived size and perceived distance in stereoscopic vision and an analysis of their causal relations. Perception \& Psychophysics, 16, 175-181.
Perrone, J. A. (1986). Anisotropic responses to motion toward and away from the eye. Perception \& Psychophysics, 39, 1-8.

Predebon, J. (1987). Familiar size and judgments of distance: Effects of response mode. Bulletin of the Psychonomic Society, 25, 244-246.

Predebon, J. (1990). Relative distance judgments of familiar and unfamiliar objects viewed under representatively natural conditions. Perception \& Psychophysics, 47, 342-348.

PREDEBON, J. (1991). Spatial judgments of exocentric extents in an openfield situation: Familiar versus unfamiliar size. Perception $\& P$ sychophysics, 50, 361-366.

PREDEBON, J. (1992a). The influence of object familiarity on magnitude estimates of apparent size. Perception, 21, 77-90.

Predebon, J. (1992b). The role of instructions and familiar size in absolute judgments of size and distance. Perception \& Psychophysics, 51, 344-345.

Predebon, J. (1993). The familiar size cue to distance and stereoscopic depth perception. Perception, 22, 985-995.

Predebon, J. (1994). Perceived size of familiar objects and the theory of off-sized perceptions. Perception \& Psychophysics, 56, 238-247.

Predebon, J., \& Wolley, J. S. (1994). The familiar-size cue to depth under reduced-cue viewing conditions. Perception, 23, 1301-1312.

Rivest, J., ONo, H., \& SAIDA, S. (1989). The roles of convergence and apparent distance in depth constancy with motion parallax. Perception \& Psychophysics, 46, 401-408.

Swanston, M. T., \& Gogel, W. C. (1986). Perceived size and motion in depth from optical expansion. Perception \& Psychophysics, 39, 309-326.

Wagner, M. (1985). The metric of visual space. Perception \& Psychophysics, 38, 483-495.

Wallach, H., \& Zuckerman, C. (1963). The constancy of stereoscopic depth. American Journal of Psychology, 76, 404-412.

\section{NOTES}

1. According to Equation $1, S_{\mathrm{S}}^{\prime} / S_{\mathrm{L}}^{\prime}$ is related to $D_{\mathrm{S}}^{\prime} / D_{\mathrm{L}}^{\prime}$ by the equation $S_{\mathrm{S}}^{\prime} / S_{\mathrm{L}}^{\prime}=\left[2 \tan \left(\theta_{\mathrm{S}} / 2\right) / 2 \tan \left(\theta_{\mathrm{L}} / 2\right)\right]\left(D_{\mathrm{S}}^{\prime} / D_{\mathrm{L}}^{\prime}\right)$. For the terminal values $\theta_{\mathrm{S}}$ and $\theta_{\mathrm{L}}$, used in the present study, if the size constancy is zero (i.e., $S_{\mathrm{S}}^{\prime} / S_{\mathrm{L}}^{\prime}=0.297$ ), $D_{\mathrm{S}}^{\prime}=D_{\mathrm{L}}^{\prime}$, and if the size constancy is perfect (i.e., $\left.S_{\mathrm{S}}^{\prime} / S_{\mathrm{L}}^{\prime}=1\right), D_{\mathrm{S}}^{\prime}=3.36 D_{\mathrm{L}}^{\prime}$. This is also the ratio of the simulated perceived depths calculated from Equation 2 (i.e., $94.5 \mathrm{~cm} / 28.1 \mathrm{~cm}$ ) using the contractions and expansions starting at the same perceived distance for perfect size constancy. The relation between $S_{\mathrm{S}}^{\prime} / S_{\mathrm{L}}^{\prime}$ and $D_{\mathrm{S}}^{\prime} / D_{\mathrm{L}}^{\prime}$, however, could not be examined in the present study because the ratio $D_{\mathrm{S}}^{\prime} / D_{\mathrm{L}}^{\prime}$ was not measured.

2. It is appropriate to call $S_{c} / S^{\prime}$ either an off-sized perception or an off-sized judgment since it has both a perceptual component $\left(S^{\prime}\right)$ and a memory component $\left(S_{c}\right)$

(Manuscript received October 14, 1996; revision accepted for publication June 25, 1997.) 